\title{
Fog Computing: análise de desempenho computacional
}

\author{
Leonardo M. Foletto ${ }^{1}$, Ivania Fischer ${ }^{2}$, Eder P. Pereira ${ }^{2}$, Roseclea D. Medina ${ }^{2}$ \\ ${ }^{1}$ Engenharia de Computação - Universidade Federal de Santa Maria \\ leonardo.folettodecomp.ufsm.br
}

${ }^{2}$ Pós-Graduação em Ciência da Computação - Universidade Federal de Santa Maria

ivaniafischer@redes.ufsm.br, epereira@inf.ufsm.br, roseclea@inf.ufsm.br

\begin{abstract}
Cloud computing aims to establish more agile communications and have a pre-processing of information. With this, in this article we will propose an analysis of the computational power of these devices and for this it presents a calculation based on hardware requirements, in order to obtain a concise metric of Fog nodes, obtaining greater precision when needing to replace some Fog node, knowing that it will perform satisfactorily in the IoT environment to which it was inserted. Each system requirement has a weight, this weight can be used to suit the most important requirements for a given environment. Fog Computing is a hot topic in the academic study [Albertin and de Moura Albertin 2017], with this still contains some points to be studied, in this article we seek to achieve results and further research on the subject in question.
\end{abstract}

Resumo. A Computação em Nuvem tem como objetivo estabelecer comunicações mais ágeis e dispor de um processamento prévio das informações. Com isto, neste artigo será proposto uma análise do poder computacional desses dispositivos e para isso apresenta-se um cálculo baseado em quesitos de hardware, para assim obter uma métrica concisa dos nodos Fog, obtendo uma maior precisão ao necessitar substituir algum nodo de Fog, sabendo que o mesmo terá um desempenho satisfatório no ambiente IoT ao qual foi inserido. Cada requesito do sistema possui um peso, este peso pode ser utilizado para adequar os requisitos mais importantes para determinado ambiente. Fog Computing é um tema em alta no estudo academico [Albertin and de Moura Albertin 2017], com isto ainda contém alguns pontos a serem estudados, neste artigo buscamos alcançar resultados e aprofundar a pesquisa no tema em questão.

keywords. Internet of Things. Fog Computing. Computational analysis.

\section{Introdução}

A Internet of Things é uma tecnologia que ao decorrer dos anos apresenta uma crescente em universidades, nas empresas e por inúmeros países [Albertin and de Moura Albertin 2017]. O desenvolvimento do estudos esses estudos é impulsionado pela facilidade de aquisição desses equipamentos, o que auxilia na disseminação da tecnologia, que nas indústrias são amplamente utilizados [Biaggi et al. 2018]. Essa disseminação é suportada por fazer-se uso da tecnologia Cloud que contém uma grande capacidade de armazenamento, porém, um ponto a salientar 
é que essa tecnologia não suporta níveis baixos de latência e pouco uso da largura de banda. Ao entorno desse contexto, surge a aquisição de uma tecnologia como solução do problema, definida como Computação em Névoa (do inglês, Fog Computing).

Pela Computação em Névoa se tratar de um processamento mais próximo aos dispositivos, é nele que concentra-se a proposta deste artigo. Dentro do contexto da Fog, esse trabalho apresenta a análise do desempenho de alguns dispositivos através de uma fórmula matemática para identificar futuramente quais dispositivos suportam a demanda de processamento necessário no ambiente da Fog. Essa fórmula irá considerar quesitos de hardware para adquirir uma classificação dos dispositivos que podem ser utilizados como nodos Fog.

Para desenvolvimento do trabalho são utilizadas as seguintes seções: a Seção 2 destaca os trabalhos relacionados encontrados na literatura, a Seção 3 argumenta sobre IoT e Fog Computing a Seção 4 apresenta os cenários de aplicação da proposta a Seção 5 apresenta a proposta da análise computacional e a Seção 6 os testes para finalizar tem-se a conclusão e as referências.

\section{Trabalhos Relacionados}

A tecnologia de Fog Computing apresenta diversos desafios [Wang et al. 2018] em segurança, regulamentação, implementação, porém IoT tem sido muito abordada, a mesma traz inúmeras melhorias em desempenho e eficiência[Coutinho et al. 2016]. Contudo, ainda assim tem-se problemáticas como segurança, gerenciamento e disponibilidade.

Obstante a isso, com o enfoque em questões de análise computacional de dispositivos, que serão Fog uma das maiores preocupações está entrelaçada a resiliência do sistema, pode-se citar [Segura et al. 2017] que dentre inúmeros objetivos de seu trabalho, apresenta a utilização de dispositivos móveis para serem utilizados como recursos computacionais, onde leva em consideração apenas duas limitações quanto sua disponibilidade computacional: bateria e mobilidade, características de processamento, armazenamento e memória são deixadas de lado, fato esse que não é muito conveniente e aplicável quando se fala de disponibilidade.

Nota-se que poucos são os estudos que objetivam focar a preocupação de questões básicas, como o funcionamento contínuo de uma Fog, sendo essas questões as que melhor auxiliam para a boa execução do ambiente. Por isso esse trabalho, em contrapartida dos apresentados, tem o enfoque em disponibilizar um ambiente mais resiliente, para isso analisa os melhores dispositivos para desempenhar o papel de uma Fog a partir de uma análise computacional dos mesmos.

\section{Internet of Things e Fog Computing}

A computação ao passar dos anos esteve focada em uma constante geração de informações, tendência essa que é crescente e é necessária que permaneça sendo utilizada, pois é esse o ponto-chave da comunicação [Albertin and de Moura Albertin 2017]. Nesse contexto, algumas aplicações necessitam de uma baixa latência para tomadas de decisões em diversos ambientes como cidades, agricultura, segurança, casas inteligentes, saúde, entre outros [Neto et al. 2017] [Geraldo Filho et al. 2018]. A Fog tem sido vista 
como a solução para alguns destes problemas, a qual é definida como uma extensão da computação em nuvem, alocada entre a Cloud e o usuário final, de modo a otimizar o processamento, armazenamento e transmissão de dados, promovendo melhorias nos processos de interação.

A IoT neste mesmo conceito de trabalho pode ser vista como uma rede de dispositivos independentes entre si, com o objetivo de coletar dados de um determinado ambiente, identificar e determinar o tipo de resposta deve-se validar para os atuadores. Porém, nestes caminhos encontram-se obstáculos como falhas em comunicações, sensibilidade a latência, entre outros. Em dispositivos que devem funcionar $24 \mathrm{~h}$ por dia, durante 7 dias da semana, alguns pontos, como os citados anteriormente, precisam ser analisados com bastante atenção para não haver lacunas a ponto de interferir na atuação de algum dispositivo.

\section{Cenário de Aplicação do Método}

Dentre os diversos cenários de aplicação de IoT [Albertin and de Moura Albertin 2017], neste artigo optou-se por um ambiente formado por três camadas, conforme Figura 1.Já para esclarecer a utilização da proposta desse artigo, focaremos em um ambiente formado por 3 camadas. No primeiro nível existem os sensores e atuadores, que são os dispositivos mais próximos dos usuários, os sensores terão o objetivo de coletar dados e enviar para as Fogs, já os atuadores, serão responsáveis por receber das Fogs comandos para exercerem alguma influência sobre o ambiente.

As Fogs serão responsáveis por receber as informações dos sensores, processar a maior parte destas informações, classificar quais devem ser enviadas para a Cloudou então, já ter uma tomada de decisão para enviar para os atuadores. A Cloud será responsável por auxiliar as Fogs em processamentos maiores, além de ser responsável pelo gerenciamento de cada Fog. Essas 3 camadas são apresentadas na Figura 1.

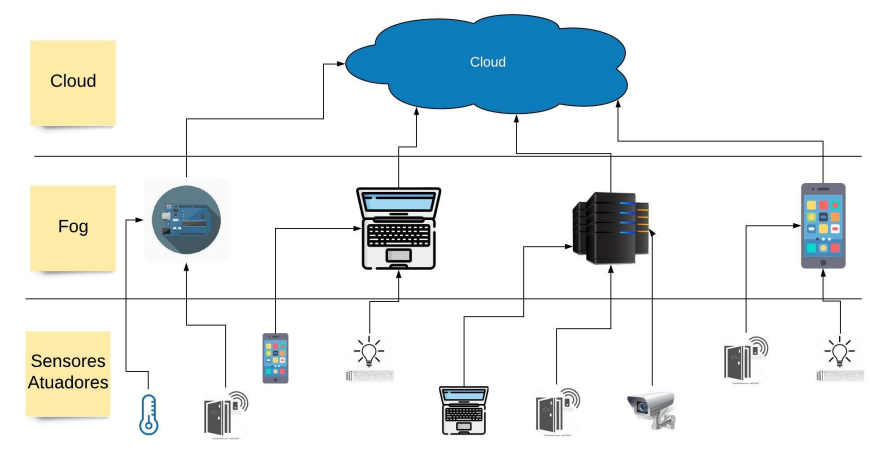

Figura 1. Ambiente loT em funcionamento normal

Considerando a topologia da Figura 1, denota-se o ambiente IoT em funcionamento sem anomalia, enquanto a Figura 2, destaca-se pelo ambiente onde ocorre a falha em um nodo Fog.

Diante do cenário da Figura 2 denota-se a importância de definir a melhor Fog para substituir a que apresentou alguma irregularidade. Por isso o trabalho propõe a análise computacional dos nodos Fog, a fim de definir a melhor Fog computacionalmente para a substituição[Pereira et al. 2018]. 


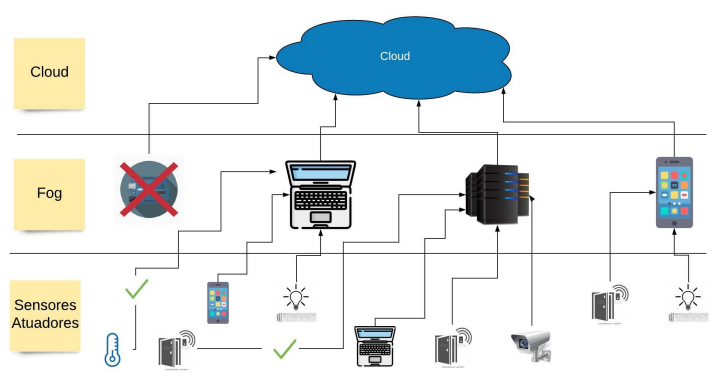

Figura 2. Ambiente loT com existência de anormalidade

\section{Proposta de Análise Computacional}

A análise computacional de desempenho refere-se ao poder que o dispositivo tem em executar determinada tarefas, ou seja, indiferentemente da quantidade de tarefas que são alocadas a ele, sua capacidade de execução será o ponto crucial para avaliação. Para realizar esta analise será realizado o uso de três recursos como importantes para o desempenho de uma Fog, sendo elas o processador, memória e disco.

Para realização desta análise foram selecionados indicadores para conseguir mensurar os níveis de desempenho computacional de cada dispositivo, a escolha destes indicadores deve-se a influência que os mesmos tem na realização de uma tarefa, sendo definido a quantidade de cores, clock e memória cache dos processadores, tempo de vida e poder de armazenamento.

Exemplificando a análise do clock de um processador que refere-se a frequência em que os processadores trabalham, caso sejam analisadas individualmente nem sempre segue a ideia de quanto maior a frequência maior o processamento para todas as informações, justamente pelas diferenças entre tarefas e ciclo de instruções de cada processador necessita para executar determinada tarefa, por isto enumeramos mais 4 indicadores para obter uma maior fidelidade nos resultados das análises.

- Core: A Figura 3 refere-se a quantidade de núcleos que estão disponíveis em um processador. No exemplo abaixo os processadores referem-se a núcleos de computadores Intel Core. Caso fossem processadores de celulares seriam denominados Octa-core, Quad-Core. Os mais utilizados atualmente são os das famílias Exynos e Snapdragon utilizados em celulares da Samsung e os da família Apple AX, utilizados em celulares da Apple.

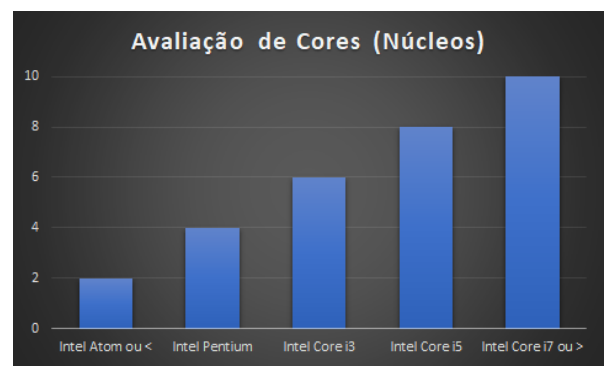

Figura 3. Núcleos dos processadores 
- Clock: Já a Figura 4 refere-se a frequência de operação dos núcleos dos processadores, ou seja, a capacidade de um processador analisar informações ao mesmo tempo. Atualmente os processadores estão trabalhando na faixa $\mathrm{MHz}$ e $\mathrm{GHz}$, porém em nossa análise consideramos processadores que trabalham na escala de $\mathrm{GHz}$.

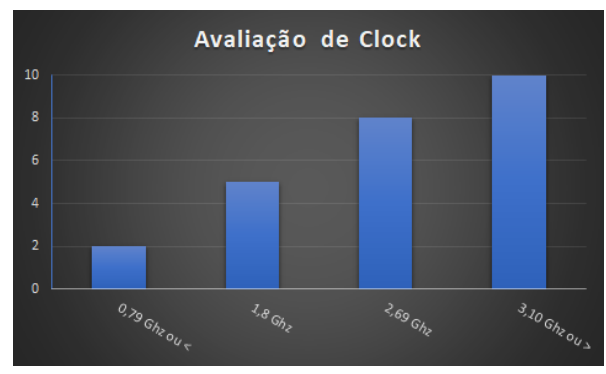

Figura 4. Frequência dos processadores

- Cache: Na Figura 5 obtemos as notas da memória cache que é um tipo de memória auxiliar, que faz diminuir o tempo de transmissão de informações entre o processador e outros componentes. Ela é utilizada na maioria das vezes em processamentos mais frequentes, visto que a velocidade de transmissão é muito superior as memórias de disco, por outro lado sua capacidade de armazenamento é muito menor ao que se obtém em discos.

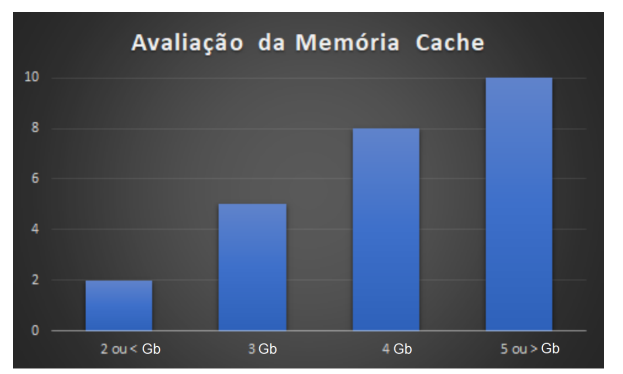

Figura 5. Memória cache dos processadores

- Tempo de Vida: Nesse indicador da Figura 6 refere-se a capacidade de duração em que os dispositivos conseguem permanecer ativos caso não estejam conectados a energia contínua, este indicador é extremamente importante mesmo nos dias de hoje, pois os sistemas não podem ter seu funcionamento comprometido em qualquer falta de energia elétrica.

- Disco: Já no desempenho de uso de um disco, como mostra na Figura 7 foi baseado na utilização de disco médio analisado por 24 horas, analisando os dispositivos em um ambiente IoT em modo ativo. Quanto maior a utilização do disco, menor o poder computacional o dispositivo tem para desempenhar novas suas funções.

As notas propostas a cada um dos indicadores não são imutáveis, porém chegou-se a estes valores a partir de testes em dispositivos sobre pressão e verificando a resposta em termos de desempenho que os dispositivos alcançavam. Colocando em prática os cálculos compostos pelos quesitos vistos acima, o modelo é formulado da seguinte maneira: 


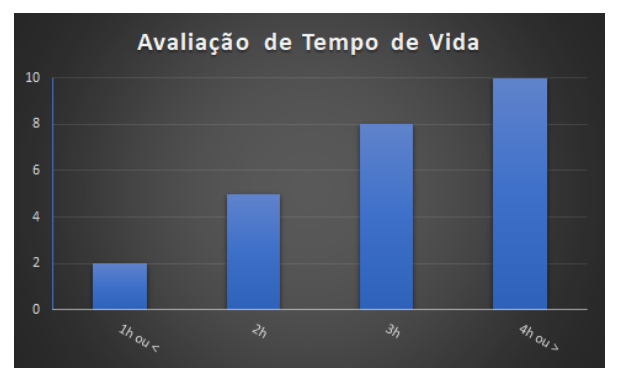

Figura 6. Tempo de vida dos dispositivos

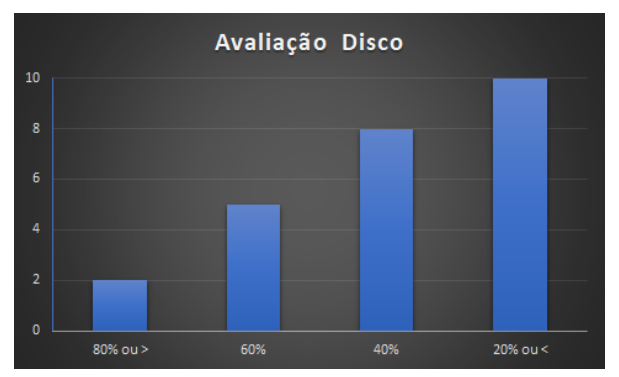

Figura 7. Utilização do disco

$$
F=[(C r * i)+(C l * i)+(T * i)+(C a * i)+(D i * i)]
$$

As siglas equivalem a: $\mathrm{F}=$ Poder Computacional; $\mathrm{i}=0.2$ (nota definida sobre o peso 10$) ; \mathrm{Cr}=$ Core $; \mathrm{Cl}=$ Clock; $\mathrm{T}=$ Tempo de Vida; $\mathrm{Ca}=\mathrm{Cache} ; \mathrm{Di}=$ Disco.

A fórmula apresentada subdivide-se em cinco indicadores igualmente balanceados, porém o peso destes indicadores poderá ser reorganizado considerando a aplicação submetida, para assim definir o dispositivo que pode ou não ser designado como uma Fog. No entanto, essa constatação surgiu da premissa de quesitos comuns que são analisados em um dispositivo, tendo o enfoque em dispositivos de ambientes IoT, considerando heterogeneidade e precisão da análise. Portanto, para destacar que o cálculo proposto é relevante e garante índices bons de precisão uma comparação com outro método de análise computacional é realizada e apresentada na próxima seção.

\section{Testes e Resultados}

Para comprovar que o cálculo proposto está dentro dos critérios gerais da análise realizada, iremos utilizar para comparação o método de análise computacional [Vazquez et al. 2002], sendo que o objetivo deste texto é apresentar um pequeno conjunto de programas que meçam o desempenho de computadores sob diversos aspectos de relevância para a química computacional e que permitam a um pesquisador acessar de forma clara índices que expressema adequação de um dado equipamento à demanda computacional doseu trabalho de pesquisa. Os testes foram realizados, tanto no modelo proposto quanto no modelo de Vasquez em um dispositivo notebook do modelo Samsung Expert X50 que contém as seguintes configurações: Processador: Intel Core ${ }^{\mathrm{TM}}$ i7 8550U (1.80 GHz até 4.0 GHz $8 \mathrm{MB}$ L3 Cache); Placa de Vídeo: NVIDIA GeForce MX110 
Graphics com 2 GB GDDR5 de memória dedicada; Memória: 8 GB DDR4; Armazenamento: 1 TB (HDD). Com isso, os resultados alcançados aplicando o modelo matemático são expressados pela Figura 8.

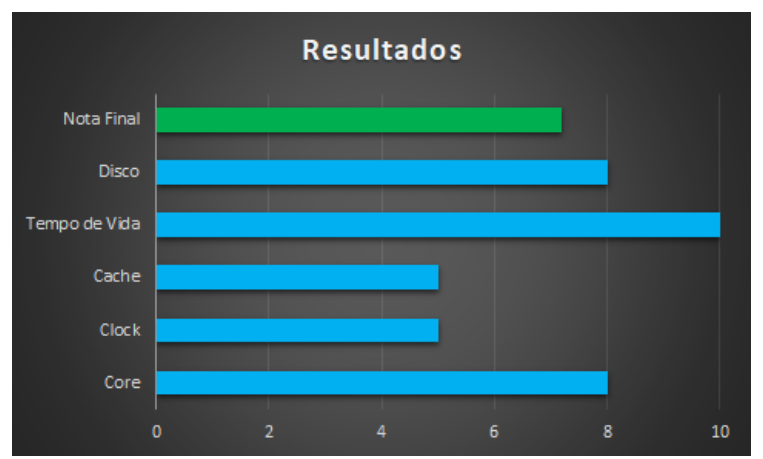

Figura 8. Resultado das recursos computacionais analisadas no notebook

Observando as notas finais do dispositivo, validamos que o modelo obteve o resultado de 7,2. Na comparação com o trabalho mencionado [Vazquez et al. 2002] leva em consideração três indicadores para obter o poder computacional.

No primeiro indicador refere-se ao desempenho de ponto flutuante, para obter estes dados foi utilizado o programa flops.c, este programa é composto por oito módulos, sendo cada módulo responsável por executar uma série de cálculos sequenciais para elevar o nível de processamento. Seu resultado é obtido na unidade de MFLOPS, a definição geral desta unidade é a capacidade do processador em realizar o maior número de operações de ponto flutuante por segundo, ao final dos testes o resultado médio em MFLOPS foi de 71,16 MBytes/s.

Já o segundo indicador refere-se ao desempenho do sistema de memória, este teste foi realizado utilizando um benchmark STREAM, que informa a velocidade que a memória atinge ao realizar uma cópia, escalonamento ou soma. O resultado obtido de cópia e também em escalonamento foi de 88,01 MBytes/s.

No último indicador a ser analisado foi o desempenho de sistema de discos, para obtermos este resultado utilizamos o script de benchmark IOzone que gera e mede uma variedade de operações de arquivos para sistemas de discos. Nele deve-se tomar um cuidado maior, atualmente a maioria dos sistemas operacionais quando um programa solicita a ele a leitura de uma parte do disco, o sistema lerá uma quantidade maior de dados para tentar antecipar uma próxima leitura do mesmo programa, já no caso de escrita, o sistema operacional retém o máximo possível de dados na memória principal antes de transferir para o disco.

Para obter um resultado o mais realístico possível deve-se utilizar arquivos de testes com tamanho igual ou superior ao tamanho da memória principal do computador para evitar falsos resultados do sistema cache. Tendo a abordagem preventiva com o cuidado os resultados alcançados foram cerca de 119 leituras e escritas resultando em uma média de valor de cerca de 53,25 leituras e escritas. Ao final desta análise foi obtido o resultado de 7,07 considerando o modelo [Vazquez et al. 2002]. Ao comparar os resultados com o modelo proposto neste artigo, cujo obteve uma nota igual a 7,2, nota-se que os resultados obtidos foram muitos próximos, alcançando o objetivo de validar o modelo matemático. 


\section{Conclusão}

Ao final deste trabalho, podemos observar que ainda existem alguns pontos a serem incluídos no modelo matemático para alcançar uma maior precisão nas aplicações, porém destaca-se a evolução do modelo proposto neste artigo ao comparar com a modelo matemático [Vazquez et al. 2002]. Atualmente existe uma exigência ainda maior do desempenho dos dispositivos, um gerenciamento muito mais criterioso e com maior precisão faz-se necessário para obter-se uma confidencialidade na execução das tarefas desejadas.

Além disso o trabalho destaca como diferencial no método proposto a preocupação com a heterogeneidade dos dispositivos. Como trabalhos futuros pretendese aplicar o método em outros dispositivos como celulares, roteadores, entre outros. Além de integrar esse cálculo a um gerenciamento proativo de Fogs a fim de adquirir números maiores de resiliência e operabilidade dos ambientes IoT.

\section{Referências}

Albertin, A. L. and de Moura Albertin, R. M. (2017). A internet das coisas irá muito além as coisas. GV EXECUTIVO, 16(2):12-17.

Biaggi, G. Q., Talhaire, T. R., Cardoso, T. C., Ramos, Y. L., de Queiroga, A. P. G., and Rodrigues, L. C. (2018). A internet das coisas como fator primordial na indústria 4.0. REVISTA ELETROONICA ENGENHARIA ESTUDOS E DEBATES, 1.

Coutinho, A., Carneiro, E. O., and Greve, F. G. P. (2016). Computação em névoa: Conceitos, aplicações e desafios. Minicursos do XXXIV SBRC, pages 266-315.

Geraldo Filho, P., Neto, J. R. T., Valejo, A., Meneguette, R. I., Villas, L. A., and Ueyama, J. (2018). Um sistema de controle neuro-fog para infraestruturas residenciais via objetos inteligentes. In Anais do XXXVI Simpósio Brasileiro de Redes de Computadores e Sistemas Distribuídos. SBC.

Neto, J. R. T., Geraldo Filho, P., Mano, L. Y., and Ueyama, J. (2017). Inca: Um sistema healthcare flexível baseado no paradigma fog computing e publish/subscribe. In Anais do I Workshop de Computação Urbana (COURB 2017), volume 1. SBC.

Pereira, E. P., Fischer, I. A., Medina, R. D., and Padoin, E. L. (2018). Smartfoglb: Arquitetura de balanceamento de carga em fog computing.

Segura, D. C. M., Stabile, R. d. S., Bruschi, S. M., and Souza, P. S. L. d. (2017). Providing computing services through mobile devices in a collaborative way-a fog computing case study. In Proceedings of the 20th ACM International Conference on Modelling, Analysis and Simulation of Wireless and Mobile Systems, pages 117-121. ACM.

Vazquez, P. A. et al. (2002). Técnicas para análise de desempenho de computadores. Química Nova.

Wang, S.-C., Tseng, S.-C., Yan, K.-Q., and Tsai, Y.-T. (2018). Reaching agreement in an integrated fog cloud iot. IEEE Access, 6:64515-64524. 